\title{
Neurological disease, emotional disorder, and disability: they are related: a study of 300 consecutive new referrals to a neurology outpatient department
}

\author{
Alan J Carson, Brigitte Ringbauer, Lesley MacKenzie, Charles Warlow, Michael Sharpe
}

Department of Psychiatry, University of Edinburgh, Royal Edinburgh Hospital, Morningside Park, Edinburgh, Scotland, UK

A J Carson

B Ringbauer

M Sharpe

Department of Medical Neurology, University of Edinburgh, Scotland, UK

C Warlow

Department of Psychological Medicine

M Sharpe

Department of Clinical Neurology, Western General Hospital, Edinburgh, Scotland, UK

L MacKenzie

Correspondence to: Dr A J Carson, Department of Psychiatry, University of Edinburgh, Royal Edinburgh Hospital, Morningside Park, Edinburgh, EH10 5HF,

Scotland, UK

email A.Carson@ed.ac.uk

Received 4 March 1999 and in revised form

19 July 1999

Accepted 5 August 1999

\begin{abstract}
Objectives-To determine the prevalence of anxiety and depressive disorders in patients referred to general neurology outpatient clinics, to compare disability and number of somatic symptoms in patients with and without emotional disorder, the relation to neurological disease, and assess the need for psychiatric treatment as perceived by patients and doctors. Methods-A prospective cohort study set in a regional neurology service in Edinburgh, Scotland. The subjects were 300 newly referred consecutive outpatients who were assessed for DSM IV anxiety and depressive disorders (PRIME-MD, and HAD), health status, and disability (SF36), and patients', GPs' and neurologists' ratings of the need for patient to receive psychiatric or psychological treatment.

Results-Of 300 new patients, 140 (47\%) met criteria for one or more DSM IV anxiety or depressive diagnosis. Major depression was the most common $(27 \%)$. A comparison of patients with and without emotional disorder showed that physical function, physical role functioning, bodily pain, and social functioning were worse in patients with emotional disorders $(p<0.0005)$. The median number of somatic symptoms was greater in patients with emotional disorders $(p<0.0005)$. These differences were independent of the presence of neurological disease. Few patients wished to receive psychiatric or psychological treatments. Both general practitioners and neurologists were more likely to recommend psychiatric treatment when the patients' symptoms were medically unexplained.
\end{abstract}

Conclusions-Almost half of new referrals to general neurology clinics met criteria for a DSM IV psychiatric diagnosis. These patients were more disabled, and had more somatic symptoms. They expressed little enthusiasm for receiving psychiatric treatment.

(F Neurol Neurosurg Psychiatry 2000;68:202-206)

Keywords: neurological disease; emotional disorder

Hospital medical services have traditionally been organised into distinct medical/surgical and psychiatric services. The medical/surgical sector offers treatment for "organic disease" and the psychiatric for "psychiatric illness". However, the limitations of this approach have become increasingly apparent with accumulating evidence that many patients would benefit from a combined approach. ${ }^{1}$

Only a few studies have examined psychiatric disorders in medical outpatients. These suggest that depression and anxiety disorders ${ }^{2}$ are not only common but also contribute to patient disability. ${ }^{3}$ We are aware of only four studies ${ }^{4-7}$ that measured the prevalence of emotional disorder (depressive and anxiety disorders) in neurology outpatient clinics. None of these examined the association of an emotional disorder with disability, nor did they assess the perceived need for treatment.

The aims of this study were to determine for new attenders at general neurological outpatient clinics in Edinburgh: the prevalence of depression and anxiety disorders; their association with patients' disability; and patients', general practitioners' (GPs') and neurologists' perception of the need for the patient to receive psychiatric/psychological treatment. (The prevalence and significance of medically unexplained symptoms in this cohort of patients will be described by us in another study.)

\section{Methods}

The study was a prospective cohort study.

NEUROLOGY OUTPATIENT SERVICES IN

EDINBURGH

Edinburgh neurology services are provided by eight consultant neurologists and their junior medical staff. All outpatient clinics take general referrals but there are additional subspecialist clinics for stroke, memory problems, and epilepsy. Each clinic sees similar patients as referrals are simply distributed by clerical staff according to available appointments. Clinic templates dictate the number of urgent, semiurgent, and routine appointments available in each clinic. Only a few patients are referred to named consultants.

\section{Sampling}

The study was conducted between November 1997 and March 1998 in five of the eight consultant outpatient clinics. Limiting the number of clinics to five allowed one clinic to be studied each weekday. All new patients were included except in one clinic where, because of the large number attending, every alternate new patient was included. 
We planned to assess a minimum of 300 patients, which we estimated would yield at least 100 with a psychiatric disorder allowing an estimate of the prevalence of specific depressive and anxiety disorders.

\section{Assessment}

New patients were sent details of the study in advance of their attendance at the clinic. After the consultation with the neurologist, patients were approached by the researcher and informed consent for participation sought. Those who agreed to participate were assessed, using a combination of structured interviews and self rating questionnaires, within 7 days of their consultation.

Patients completed the self rated hospital anxiety and depression scale (HAD) ${ }^{8}$ designed for use in patients with comorbid medical conditions. Psychiatric diagnoses, according to the DSM IV classification, ${ }^{9}$ were made using the primary care evaluation of mental disorders (PRIME-MD), ${ }^{10}$ a brief structured interview of proved reliability and validity. All subjects completed the diagnostic interview in full. We also asked subjects to complete the PRIME-MD questionnaire but did not use it as a screening instrument. The specific diagnoses included in the category "emotional disorder" were major depression, minor depression (research criteria, appendix B, DSM IV), dysthymia, panic disorder, generalised anxiety disorder, and anxiety disorder not otherwise specified.

Patients' perceptions of disability were measured using the medical outcome study scale, 36 item short form (SF-36). ${ }^{11}$ This self rated scale contains eight subscales: general health perceptions, physical functioning, physical role functioning, bodily pain, social functioning, vitality, mental health, and emotional role functioning.

The number of somatic symptoms the patient reported were measured using a checklist of the 15 most common symptoms, excluding upper respiratory tract infections, which patients report in primary care. ${ }^{12}{ }^{13}$ The checklist is part of the patient questionnaire of the PRIME-MD.

Patients' perception of need for any form of psychiatric/psychological treatment was recorded by a simple yes or no checklist before and after their consultation. General practitioners made a similar rating before the patients attending the neurologists. The neurologists made their rating after the consultation.

Assessments were carried out by AC or BR, both of whom had been trained in the use of the assessment instruments. At the time of assessment researchers were blind to the neurologists' and general practitioners' assessments. A sample of 50 of BR's interviews were tape recorded and rerated blind by AC to check reliability. Only one case was categorised as having a psychiatric disorder by BR when in the opinion of AC they failed to meet criteria.

In addition to the above assessments, the consulting neurologist rated the extent to which the patient's symptoms could be ex- plained by neurological disease ("organicity") on a four point Likert scale ("not at all explained", "somewhat explained", "largely explained", or "completely explained"). These ratings were reviewed after 6 months by case note review to determine whether they were changed by investigations or further clinical developments. Final scores were dichotomised into "neurological disease" ("largely explained" and "completely explained") and "unexplained symptoms" ("not at all explained" and "somewhat explained"). The results from this are reported in full ${ }^{14}$ but are used to aid the analysis of data in this study.

\section{Analysis}

The HAD was scored to produce both separate depression and anxiety ratings and also a total "emotional distress" score. The PRIME-MD was used to generate individual DSM-IV diagnoses. All anxiety and depressive diagnoses were then combined to produce a category of emotional disorder used in further analysis. The SF-36 scale scales were converted to percentages as recommended by the authors. In all domains of the SF-36 a lower functioning score indicates increased disability/pain.

Associations between emotional disorders and disability were examined as follows: firstly, we examined correlations between the continuous scale of severity of distress (HAD) and SF-36 scores. Secondly, we compared SF-36 subscale scores of those with a diagnosis of emotional disorder and those without. Then, to test the hypothesis that any association between emotional disorder and disability was due to neurological disease we further divided the two subsamples described above (those with a diagnosis of emotional disorder and those without) according to the presence or absence of neurological disease. A comparison of disability ratings across the four groups was then made. The data were not normally distributed so the Kruskal-Wallis test was used. Confidence intervals were calculated using non-parametric techniques and tables.

The number of somatic symptoms reported by the patient and need for psychiatric/ psychological treatment as perceived by patient, GP, and neurologist were analysed in a similar fashion.

\section{Results}

Of 364 new patients booked to attend the designated clinics during the study period $48 \mathrm{did}$ not attend their appointments, leaving 316 eli-

Table 1 Diagnoses given to illustrate case mix $n=300^{*}$

\begin{tabular}{ll}
\hline Headache & $63(21 \%)$ \\
Epilepsy/fits/pseudoseizures & $43(14 \%)$ \\
Multiple sclerosis & $30(10 \%)$ \\
Neuropathies (peripheral/entrapment) & $25(8 \%)$ \\
Syncope & $22(7 \%)$ \\
Spinal pathology (cervical/lumbar) & $22(7 \%)$ \\
'Dizziness' & $8(3 \%)$ \\
Parkinson's disease & $7(2 \%)$ \\
Psychiatric diagnosis only & $7(2 \%)$ \\
Other† & $73(26 \%)$
\end{tabular}

* Diagnoses do not necessarily indicate neurological disease; some are simply symptom descriptions.

† Diagnoses in the "other category" each had a frequency of less than $2 \%$. 
Table 2 Psychiatric diagnoses showing number of patients (and percentage) in each diagnostic category $(n=300)$ *

\begin{tabular}{ll}
\hline DSM IV Diagnosis & $n(\%, 95 \%$ CI $)$ \\
\hline Major depression & $77(26,21-31)$ \\
Minor depression $\dagger$ & $23(8,5-11)$ \\
Dysthymia & $46(15,11-19)$ \\
Panic disorder & $21(7,4-10)$ \\
Generalised anxiety disorder & $28(9,6-13)$ \\
Anxiety disorder NOS $\ddagger$ & $53(18,13-22)$ \\
\hline
\end{tabular}

${ }^{\star} 67(22 \%)$ patients met criteria for more than one disorder. † Minor depression was diagnosed according to DSM IV research criteria.

$\ddagger$ Not otherwise specified.

gible to participate. Of these, 12 refused to participate, one was too cognitively impaired to be assessable, two patients disappeared and were untraceable, and one patient found the assessment questions distressing and withdrew. This left 300 patients, a participation rate of $96 \%$ of attenders and $82 \%$ of all those referred.

One hundred and seventy four $(58 \%)$ of the patients were female. The mean age was 43 (SD 16.2) years with a range from 14 to 88 years. Table 1 describes the neurologists' final "diagnoses" of patients in the sample. In some cases the "diagnoses" are merely symptom descriptions.

The median HAD total score for the sample was 10 , with a median HAD depression score of 3 and anxiety score of 6 . Criteria for one or more DSMIV diagnoses of emotional disorder were met by $140(47 \%)$ of patients (table 2). Sixty seven patients met criteria for more than one disorder, in 58 cases this was major depression plus another disorder.

Table 3 shows correlations between the HAD scores (emotional distress) and the SF-36 scores (disability). The HAD depression score was more closely associated with disability, particularly in physical function and role functioning physical domains, than the HAD anxiety score.

The main finding was that patients with emotional disorders were substantially more disabled on all eight subscales of the SF-36 (table 4). Four of the subscales of the SF-36, general health perceptions, vitality, emotional role functioning, and mental health, measure symptoms that substantively overlap with those recorded by the PRIME-MD and the HAD scale. We will not comment further on scores from these four subscales as they add no meaningful data. (However, interested readers are invited to contact the senior author for full details). Furthermore, subdivision of the sample into those with neurological disease (210 $(70 \%))$, and those with unexplained symptoms (90 (30\%); see table 5) indicated that this association with emotional disorder was independent of the presence of neurological disease.

An analysis of the associations between emotional disorder and number of somatic symptoms showed that patients with an emotional disorder reported twice as many somatic symptoms as patients without an emotional disorder. Again, independent of the presence of neurological disease (tables 3, 4, 5).

We did not examine the associations between individual emotional disorder diagnoses and disability for two reasons. Firstly, there would be small numbers in individual cells increasing the likelihood of errors. Secondly, about one third of patients with emotional disorders had more than one diagnosis.

Only $11 \%$ of the 140 patients with an emotional disorder expressed any perceived need for some form of psychological/ psychiatric treatment. Both GPs and neurologists perceived a greater need for such treatment but were more likely to recommend this in cases where the patient had unexplained symptoms, irrespective of the presence of emotional disorders (table 6).

\section{Discussion}

Almost half of new attenders at neurology clinics in Edinburgh had an emotional (anxiety or depressive) disorder. The most common diagnostic category was major depression (26\%). These emotional disorders were strongly associated with increased disability, more pain, and

Table 3 Correlations between scores on the HAD scale, and scores on the SF-36 subscales and reported number of somatic symptoms ( $n=300)$

\begin{tabular}{|c|c|c|c|c|c|c|c|c|}
\hline & \multirow[b]{2}{*}{$\begin{array}{l}\text { No of somatic } \\
\text { symptoms }\end{array}$} & \multicolumn{4}{|l|}{$S F-36$} & \multirow[b]{2}{*}{$\begin{array}{l}\text { HAD } \\
\text { total score }\end{array}$} & \multirow[b]{2}{*}{$\begin{array}{l}\text { HAD } \\
\text { depression score }\end{array}$} & \multirow[b]{2}{*}{$\begin{array}{l}\text { HAD } \\
\text { anxiety score }\end{array}$} \\
\hline & & $\begin{array}{l}\text { Physical } \\
\text { function }\end{array}$ & $\begin{array}{l}\text { Physical role } \\
\text { function }\end{array}$ & Bodily pain & $\begin{array}{l}\text { Social } \\
\text { function }\end{array}$ & & & \\
\hline HAD total score & 0.5 & -0.3 & -0.4 & -0.4 & -0.6 & - & 0.8 & 0.9 \\
\hline HAD depression score & 0.4 & -0.5 & -0.4 & -0.4 & -0.5 & 0.8 & - & 0.6 \\
\hline HAD anxiety score & 0.4 & -0.2 & -0.2 & -0.3 & -0.4 & 0.9 & 0.6 & - \\
\hline
\end{tabular}

All the correlations were statistically significant $(\mathrm{p}<0.0005$, two tailed).

In the SF36 lower scores indicate increased disability or pain, hence the negative correlations between HAD and SF36 scores.

Table 4 Relation between emotional disorder, demographics, HAD score, disability, pain, and the number of somatic symptoms. ( $n=300)$

\begin{tabular}{|c|c|c|c|c|}
\hline & Emotional disorder $n=140$ & No emotional disorder $n=160$ & Mann-Whitney $U$ & $p$ Value \\
\hline No male $(\%, 95 \%$ CI $)$ & $50(36,28-44)$ & $76(48,40-55)$ & $4.3^{\star}$ & 0.04 \\
\hline Median $(95 \% \mathrm{CI})$ age & $41(38-44)$ & $42(38-44)$ & 10670 & 0.7 \\
\hline Median $(95 \% \mathrm{CI})$ total HAD score & $17(14-19)$ & $6(6-8)$ & 2374 & $<0.0005$ \\
\hline \multicolumn{5}{|l|}{ Median (95\% CI) SF36 scoret: } \\
\hline Physical function & $70(60-80)$ & $90(90-95)$ & 7431 & $<0.0005$ \\
\hline Physical role functioning & $25(0-50)$ & $100(75-100)$ & 6658 & $<0.0005$ \\
\hline Bodily pain & $51(42-52)$ & $80(72-84)$ & 5115 & $<0.0005$ \\
\hline Social functioning & $62.5(50-62.5)$ & $100(100-100)$ & 6914 & $<0.0005$ \\
\hline Median No $(95 \%$ CI) somatic symptoms & $6(5-7)$ & $3(2-3)$ & 5104 & $<0.0005$ \\
\hline
\end{tabular}

${ }^{\star} \chi^{2}$ test.

† Lower scores on the SF-36 equate with increased disability or pain; all scores range $0-100$. 
Table 5 Relation between emotional disorder, demographics, total HAD score, disability, pain, and somatic symptoms showing the effect of subdividing the sample according to the presence or absence of "organic" neurological disease ( $n=300)$

\begin{tabular}{|c|c|c|c|c|c|c|}
\hline & \multicolumn{2}{|l|}{ Emotional disorder } & \multicolumn{2}{|c|}{ No emotional disorder } & \multirow[b]{2}{*}{$\chi^{2} \ddagger$} & \multirow[b]{2}{*}{$p$ Value } \\
\hline & $\begin{array}{l}\text { Unexplained } \\
\text { symptoms } n=60\end{array}$ & $\begin{array}{l}\text { Neurological } \\
\text { disease } n=80\end{array}$ & $\begin{array}{l}\text { Unexplained } \\
\text { symptoms } n=30\end{array}$ & $\begin{array}{l}\text { Neurological disease } \\
n=130\end{array}$ & & \\
\hline No male $(\%, 95 \% \mathrm{CI})$ & $23(38,26-52)$ & $27(34,24-45)$ & $10(33,17-53)$ & $66(51,42-59)$ & 7.2 & 0.07 \\
\hline Median $(95 \% \mathrm{CI})$ age & $38(36-41)$ & $43(40-51)$ & $43(38-47)$ & $40(37-44)$ & 3.7 & 0.3 \\
\hline Median ( $95 \% \mathrm{CI}$ ) total HAD score & $17.5(14-19)$ & $16(12-20)$ & $7.5(4-10)$ & $6(5-7)$ & 140 & $<0.0005$ \\
\hline \multicolumn{7}{|l|}{ Median (95\% CI) scores SF-36\$: } \\
\hline Physical function & $72.5(65-85)^{\star}$ & $60\left(50-70^{\star}\right.$ & $90(75-100) \dagger$ & $90(85-95) \dagger$ & 27.8 & $<0.0005$ \\
\hline Physical role functioning & $25(0-50)^{\star}$ & $25(0-50)^{\star}$ & $100(75-100) \dagger$ & $100(75-100) \dagger$ & 41.2 & $<0.0005$ \\
\hline Bodily pain & $41(30-50)^{\star}$ & $52(40-60)^{\star}$ & $68(52-84) \dagger$ & $84(72-84) \dagger$ & 37.5 & $<0.0005$ \\
\hline Social function & $50(37.5-62.5)^{\star}$ & $62.5(50-75)^{\star}$ & $100(62.5-100) \dagger$ & $100(100-100) \dagger$ & 72.3 & $<0.0005$ \\
\hline $\begin{array}{l}\text { Median No }(95 \% \mathrm{CI}) \text { somatic } \\
\text { symptoms }\end{array}$ & $6(5-7)^{\star}$ & $6(5-6)^{\star}$ & $3(2-5) \dagger$ & $3(2-3) \dagger$ & 70.2 & $<0.0005$ \\
\hline
\end{tabular}

^Between group comparisons using Mann-Whitney U test all non-significant ( $p>0.05)$.

† Between group comparisons using Mann-Whitney U test all non-significant ( $p>0.05)$.

$\ddagger$ Kruskall-Wallis test, 3 df.

$\S$ A lower score on the SF 36 equates with increased disability: all subscales range from 0-100.

Table 6 Number of patients requiring psychiatric/psychological treatment as perceived by the patients, the GPs, and the neurologist $(n=300)$ (\% of total number in each column, 95\% CI)

\begin{tabular}{llllll}
\hline & \multicolumn{2}{l}{ Emotional disorder } & & & No emotional disorder \\
\cline { 2 - 3 } \cline { 5 - 6 } & $\begin{array}{l}\text { Unexplained symptoms } \\
n=60\end{array}$ & $\begin{array}{l}\text { Neurological disease } \\
n=80\end{array}$ & & $\begin{array}{l}\text { Unexplained symptoms } \\
n=30\end{array}$ & $\begin{array}{l}\text { Neurological disease } \\
n=130\end{array}$ \\
\hline Patients preconsultation & $7(12,5-23)$ & $5(7,2-15)$ & & $0(0,0-12)$ & $1(1,1-4)$ \\
Patients postconsultation & $8(14,6-25)$ & $7(9,4-18)$ & & $0(0,0-12)$ & $1(1,1-4)$ \\
GPs & $20(34,22-47)$ & $9(12,6-22)$ & & $9(31,15-51)$ & $13(10,6-16)$ \\
Neurologists & $33(56,42-69)$ & $9(12,6-22)$ & & $7(24,10-44)$ & $3(2,1-7)$ \\
\hline
\end{tabular}

more somatic symptoms. This association was present whether analysed using a continuous variable (the HAD score) or a categorical approach (the PRIME-MD produced DSM IV diagnoses). On the HAD scale the relation was strongest for depressive symptoms. The association of disability and emotional disorder was equally strong in those with neurological disease and those with unexplained symptoms.

It is of interest to note that despite the apparent importance of emotional disorders, few of these patients expressed any interest in psychological/psychiatric treatment and rather expressed their preference for use of the traditional medical approach. There was a discrepancy between patients and their doctors in that the neurologists and GPs were more likely to consider psychiatric/psychological treatment to be appropriate. However, they were more likely to suggest this for those patients with unexplained symptoms rather than those with neurological disease and comorbid emotional disorder.

When interpreting results the representativeness of the sample must be considered. The participating clinics were representative of the overall service and referrals were taken from a common pool. However certain specialised clinics, in particular neurovascular and memory clinics, are not included and potentially render our sample unrepresentative of patients seen in less specialised services. Nonetheless, the very high participation rate $(96 \%)$ indicated that this sample is representative of new attenders to the Edinburgh general neurology clinics.

The PRIME-MD is based on the structured clinical interview for DSM III- $\mathrm{R}^{15}$ and has been tested extensively in four American primary care settings, ${ }^{10}$ three of which were hospital based clinics and one was a family based clinic.
The overwhelming majority of patients in the original sampling frame had a comorbid physical disorder. In this patient group it was specific for the major diagnostic categories; $92 \%$ for any mood disorder, $98 \%$ for major depression, $96 \%$ for dysthymia, $99 \%$ for panic disorder, and $97 \%$ for generalised anxiety disorder. Its weakness was sensitivity, with a detection rate of $67 \%$ for any mood disorder and $83 \%$ for any psychiatric condition. We tried to improve on this sensitivity by asking all the questions from each module in the interview schedule, as it had been originally designed, and omitted the screening questionnaire that was later developed to decrease the time spent in completing the interview. If it remained insensitive, this will have resulted in an underestimate of psychiatric morbidity. An examination of the difference in median total HAD scores, in this sample, between those identified by the PRIME-MD as having an emotional disorder (median 17) and those who do not (median 6), demonstrated a difference of 11 points, providing further reassurance of the validity of the PRIME-MD in patients with comorbid physical illness.

In accordance with the authors' instructions we converted the raw SF-36 scores into percentages. It should be noted that although making the findings easier to interpret, this conversion can give the appearance that some domains, particularly the role functioning domains, have a greater range of scores than they actually do. For example, in the physical role functioning domain, the raw scores range from 4 to 8 , and converted they range from 0 to 100.

It may be asked whether the emotional disorders identified persist. Follow up studies of inpatient populations have found that such disorders often remit after discharge. ${ }^{16}$ The suggested explanation is that the emotional 
disorder reflects the stress of a stay in hospital. This hypothesis may be less applicable to outpatients. We are, however, following up this cohort and will report on this separately.

There have been only four previous studies of anxiety and depression in neurology outpatient clinics, one in the north east of England, ${ }^{4}$ two in the United States, ${ }^{5}$ and one in the Netherlands. ${ }^{6}$ Two ${ }^{45}$ were carried out using self rating scales, the general health questionnaire, ${ }^{17}$ and the symptom check list- $90 .{ }^{18}$ In these studies the prevalence of patients with emotional disorder was $27 \%$ and $51 \%$ respectively. Neither scale allowed for the generation of individual diagnoses, but were limited to the use of cut offs to describe a patient as "likely" to have a emotional disorder. The Dutch study used a diagnostic interview, the present state examination (PSE), ${ }^{19}$ and found that $25 \%$ of new patients met PSE criteria for an emotional diagnosis, with depressive disorders (15\%) being most common. The final study ${ }^{7}$ relied on the clinical impression of a neurology resident and reports a much lower rate of depression. None of these studies commented on disability or the perception of treatment need.

The results from our study are of particular interest because they demonstrated a strong relation between emotional disorders and disability. In the study of Wells et al, ${ }^{3}$ they also found that the combined presence of depression and a medical condition had a magnified effect on disability. Our findings replicate this in patients with neurological diseases, a group not included in the study of Wells et al. Of particular interest is the finding that a similar effect exists in patients with unexplained symptoms.

What should be done? The important questions now are firstly, do these emotional disorders persist? Secondly, can they be successfully treated? Thirdly, does successful treatment of an emotional disorder lead to reduction in the patient's disability? It should be noted however, that whereas the patients' doctors were of the view that many of the patients should receive psychiatric or psychological treatment, by and large the patients themselves were not. This has important implications for how psychiatric treatments are "sold" to these patients. If it can be shown that effective treatment can be provided then it seems likely that the first part of any treatment strategy will involve some form of symptom reattribution, possibly using some of the techniques of cognitive therapy. It may be that future neurologists will have to be conversant in the basics of these techniques.

\section{Conclusions}

Almost half of the new referrals to neurology outpatient clinics met criteria for a DSM IV mood or anxiety disorder. These patients had greater disability than those without an emotional disorder irrespective of whether they had neurological disease or unexplained symptoms. Despite this, few patients expressed enthusiasm for psychiatric treatment. Both general practitioners and neurologists were more likely to recommend psychiatric treatment when the patients' symptoms were medically unexplained.

The study was funded by the University of Edinburgh.

1 Royal Colleges of Physicians and Royal College of Psychiatrists. Foint working party report: the psychological care of medical patients; recognition of need and service provision. London: Royal College of Physicians, 1995.

2 Hamilton J, Campos R, Creed F. Anxiety, depression and the management of medically unexplained symptoms in medical clinics. F R Coll Physicians Lond 1996;30:18-20.

3 Wells KB, Stewart A, Hays RD, et al. The functioning and well-being of depressed patients. $\mathscr{f} A M A$ 1989;262:914-19.

4 Kirk C, Saunders M. Primary psychiatric illness in a neurological out-patient department in north east England. Acta Psychiatr Scand 1977;56:294-302.

5 Berlin RM, Ronthal M, Bixler EO, et al. Psychiatric symptomatology in an outpatient neurology clinic. f Clin Psychiatry 1983;44:204-6.

6 Van Hemert AM, Hengeveld MW, Bolk JH, et al. Psychiatric disorders in relation to medical illness among patients of a general medical out-patient clinic. Psychol Med 1993;23: $167-73$.

7 Shiffer RB. Psychiatric aspects of clinical neurology. Am $\mathcal{F}$ Psychiatry 1983;140:205-7.

8 Zigmond AS, Snaith RP. The hospital anxiety and depression scale. Acta Psychiatr Scand 1983;67:361-70.

9 American Psychiatric Association. Diagnostic and statistical manual of mental disorders, 4th edition. Washington, DC: American Psychiatric Association, 1994.

10 Spitzer RL, Williams JB, Kroenke K, et al. Utility of a new procedure for diagnosing mental disorders in primary care. The PRIME-MD 1000 study. FAMA 1994;272:1749-56.

11 Ware JE, Sherbourne CD. The MOS 36 item short form health survey (SF-36). Conceptual framework and item selection. Med Care 1992;30:473-83.

12 Kroenke K, Arrington ME, Mangelsdorff AD. The prevalence of symptoms in medical outpatients and the adequacy of therapy. Arch Intern Med 1990;150:1685-9.

13 Schappert SM. National Ambulatory Medical Care Survey. Vital Health Stat 1989;13:110:2-11.

14 Carson AJ, Ringbauer B, Stone J, et al. Do medically unexplained symptoms matter? A prospective cohort study of 300 new referrals to neurology outpatient clinics. F Neurol Neurosurg Psychiatry 2000;68:000-000.

15 Spitzer RL, Williams JBW, Gibbon M, et al. The structured clinical interview for DSMIII-R (SCID), 1: history, rational and description. Arch Gen Psychiatry 1992;49:624-9.

16 Mayou RA, Hawton KE. Psychiatric disorder in the general hospital. Br F Psychiatry 1986;149:172-90.

17 Goldberg D. The detection of psychiatric illness by questionnaire. Maudsley Monograph 21. London: Oxford University Press, 1972.

18 Derogatis LR. SCL-90 Administration, scoring and procedures manual I. Baltimore, MD: John Hopkins University, 1977.

19 Wing JK, Cooper J, Sartorius N. The measurement and classification of psychiatric symptoms. Cambridge: Cambridge University Press, 1974. 